\title{
Electrochemical Noise Measurement and Analysis of Hydrogen-air PEM Fuel Cell
}

\author{
Astafev EA ${ }^{1 *}$, Dobrovolsky YA 1 , Ukshe $\mathrm{AE}^{1}$, Manzhos RA ${ }^{1}$ and Grafov $\mathrm{BM}^{2}$ \\ ${ }^{1}$ Institute of Problems of Chemical Physics RAS, Chernogolovka, Moscow Region, Russia \\ ${ }^{2}$ AN Frumkin Institute of Physical Chemistry and Electrochemistry RAS, Leninskii Prospekt 31, Moscow, Russia
}

\begin{abstract}
The method of measurement and analysis of electrochemical noise was used to test a hydrogen-air polymer electrolyte fuel cell and its electro catalytic electrodes. The measurement was carried out during stepped electrochemical aging. After each aging step, the electrochemical noise was measured and analyzed. Besides, power characteristics of the fuel cell and its impedance spectrums were collected to characterize fuel cell at different stages of aging. These data were compared with the electrochemical noise analysis results. Experimental study of electrochemical noise of PEM fuel cell electrodes in a liquid electrolyte during artificial degradation in solutions saturated by various gases was carried out. A decrease in activity due to the degradation of the electrodes was showed to be accompanied by a decrease in the integral of noise amplitude and a significantly lower dependence of frequency exponent (and associated Hurst exponent). At the first stage of noise analysis, a baseline was subtracted from the initial signal. Various approaches for baseline determination were used, namely polynomial approximation and the method of moving average. At the next stage of noise analysis, a Fourier transform was applied. A decrease in magnitude of peaks for some characteristic frequencies was shown to correlate with the decrease in load currents in the course of fuel cell aging.
\end{abstract}

Keywords: Electrochemical noise; Fuel cell; Fourier transform; Degradation

\section{Introduction}

Electrochemical power sources are widely used in our life. They have got a lot of advantages. They are very useful and sometimes irreplaceable. Nevertheless, they possess some disadvantages, the main of which is the limited lifetime. So we have to test them to predict their remaining lifetime, in order to prevent such problems as critical power loss and self destruction which is possible at the end of their lifetime.

There are several methods and procedures to test electrochemical power sources:

(i) The measurement of open circuit potential, (ii) the impedance and (iii) pulse methods $[1,2]$. All of them have heir advantages and disadvantages. Nevertheless, a development of new method not only for power sources testing but also for other electrochemical needs (e.g., improvement of the electrochemical impedance method) would of great use.

An electrochemical noise measurement and analysis can meet these requirements. This method is widely used in corrosion measurements nowadays [3]. It is quite a fast and express method compared to other techniques. It is a well-automated method. Noise analysis is widely used for other non-electrochemical applications, and good theoretical and mathematical fundaments were developed for it $[4,5]$.

Some difficulties and problems arise if one applies an electrochemical noise measurement method to test electrochemical power sources. The first problem is a technical one of accurate measurement of small electrical signals as micro volts on the background of higher voltages as volts. Secondly, this method requires a complicated analysis both on the stage of baseline and drift elimination and on the stage of actual noise analysis [6,7]. The third problem is a high sensitivity of electrochemical noise to such factors as sample history and potential drift [8]. Therefore, non-electrochemical effects should be eliminated. At least the measurement equipment should possess low level of selfnoise and the sample itself should be carefully shielded.
The current work is concerned with noise measurement and analysis for hydrogen-air polymer electrolyte membrane (PEM) fuel cell (FC). Moreover, another challenge of this study is to obtain curtain parameters of noise signal that correspond to a degree of fuel cell degradation during its life cycle.

Importantly, all of the measurements were held only at open circuit potential (OCP). The reason for this is a fact that powerful fuel cell is not always possible to be tested at some load current as a test equipment should be very large and powerful to make such loads. The apparatus for noise measurement can be small by design both for micro fuel cell and for a powerful power source based on a kilowatts fuel cell. That is why impedance spectra for loaded fuel cell were not measured in this work. Besides, we have experienced in such measurements earlier $[1,2]$. Therefore, the impedance was measured only at OCP, and both methods were realized in conditions in which they are to be used in production for real fuel cell stacks.

In the current work, the degradation mechanisms were not analyzed. Thus to a first approximation, a pure phenomenological approach was used. A search for a correlation between the change in the spectrum of low-frequency flicker noise (both common characteristics and amplitude of the individual resonance peaks) and fuel cell degradation degree was the object of the investigation. In addition, besides the membrane electrode assembly (MEA) itself the noise of individual electrodes in liquid electrochemical cell was investigated in

*Corresponding author: Astafev EA, Institute of Problems of Chemical Physics RAS, Chernogolovka, Moscow Region, Russia, Tel: +74965221657; E-mail: tdsipch@list.ru

Received November 27, 2016; Accepted November 30, 2016; Published December 03, 2016

Citation: Astafev EA, Dobrovolsky YA, Ukshe AE, Manzhos RA, Grafov BM (2016) Electrochemical Noise Measurement and Analysis of Hydrogen-air PEM Fuel Cell. J Adv Chem Eng 6: 162. doi: 10.4172/2090-4568.1000162

Copyright: (c) 2016 Astafev EA, et al. This is an open-access article distributed under the terms of the Creative Commons Attribution License, which permits unrestricted use, distribution, and reproduction in any medium, provided the original author and source are credited. 
control experiments.

\section{Materials and Methods}

\section{Materials}

The laboratory samples of polymer electrolyte fuel cell were tested. The measurements were carried out with a Electrochem PEM fuel cell test kit with operation area of $1 \mathrm{~cm}^{2}$. MEAs were assembled using the most general technique of hot pressing of electrodes with the polymer membrane [1]. The membrane was Nafion NRE-212. The electrodes were prepared on the basis of Toray gas diffusion carbon paper and $\mathrm{E}^{-T_{E K}}\left(10 \%\right.$ Pt on Vulcan ${ }^{\circ} \mathrm{XC}-72$ carbon black) as the catalyst. Same electrodes were utilized for control measurements in liquid electrolyte cell.

Hydrogen and air were used as the working gases for test cell. Hydrogen was obtained by means of a commercial generator of pure hydrogen Tsvet chrome Ltd. The air was supplied by an air pump. All gases were humidified up to $100 \%$ of relative humidity at room temperature.

\section{Electrochemical measurements}

The procedure of forced degradation (repeated oxidation-reduction cycles) was used [2]. Each sample of a fuel cell was electrochemically aged by potential cycling in a wide range (from $-1 \mathrm{~V}$ to $1 \mathrm{~V}$ ) for 30 cycles. Each aging stage was applied 8 times so a set of subsequent aging stages was obtained.

The load characteristics of a fuel cell were collected by voltage cycling from open circuit potential $(c a 1 \mathrm{~V})$ to $0.4 \mathrm{~V}$ with the scan rate of $0.05 \mathrm{~V} / \mathrm{s}$. Current at the $0.4 \mathrm{~V}$ on the 4 th cycle was used for comparison with the results of noise analysis.

Separate electrodes were aged by voltage cycling in a potential range from -0.035 to $1.7 \mathrm{~V}$ (with respect to NHE) with scan rate of 0.05 $\mathrm{V} / \mathrm{s}$ for 25 cycles. The degradation procedure and other electrochemical measurements were carried out in a glass cell in vacuum de-aerated 0.5 $\mathrm{M}$ sulfuric acid. As the main idea in these experiments, two identical electrodes were used for all operations. A state of electrode surface was controlled by SEM, but there was no visual difference in the images obtained before and after overall aging procedure.

The electrochemical impedance was measured in a range from 30 $\mathrm{kHz}$ to $0.1 \mathrm{~Hz}$ at open circuit voltage of a fuel cell with AC amplitude of $0.005 \mathrm{~V}$. After load treatment the fuel cell was put in a 5 minutes relaxation before noise and impedance measurements. From electrochemical impedance measurements, some characteristics of fuel cell such as electrochemical reaction resistance $R_{f}$ were obtained and compared with the results of electrochemical noise analysis.

The measurement of electrochemical noise was carried out for an unloaded fuel cell supplied with hydrogen and air in operation mode. The value of open circuit potential was close to $1 \mathrm{~V}$. For every sample experiment was repeated for 3 to 7 times to achieve reproducibility and eliminate accidental deviations in experiment.

All conventional electrochemical experiments such as voltage cycling and impedance measurements were carried out using Elins P-50X potentiostat-galvanostat supplied with FRA module. Electrochemical noise was measured by means of a custom precision instrument designed by Elins Ltd. Its input voltage range varies from -4 to $4 \mathrm{~V}$, resolution of analog to digital converter is more than 24 bits, sample rate is up to 10 points per second.

\section{Noise analysis}

A spectral density of the noise was calculated in order to analise it. The spectra were calculated as the real component of Fourier image of the correlation function. That means a cosine transformation $S(f)$ (where $f$ is a frequency) defined as:

$$
S(f)=\int_{-\frac{T}{2}}^{\frac{T}{2}}(V(t) V(t+x)(\cos (2 \pi f x) d x,
$$

Where $\langle V(t)>=0$.

The power spectrum $S(f)$ of flicker $(1 / f)$ noise is known to be divided into two zones:

$$
\begin{aligned}
& 1 / f^{n}, \text { if } f>>f_{0} \\
& S(f)= \\
& S(0), \text { if } f<<f_{0},
\end{aligned} \quad\{
$$

where $f_{0}=1 / 2 \pi t_{0}$ is the inverse value of correlation time.

To determine parameters that probably will indicate the degradation degree, a resonant peak at high frequency was compared with degradation degree. Moreover, the growth speed of spectral power of flicker-noise with the decrease in frequency that is the exponent $n$ in (2) was analyzed. Thus, a decrease in $2.5 \mathrm{~Hz}$ resonant peak amplitude showed a good correlation with the degree of fuel cell degradation. For separate electrodes, $n$ value was selected as a second phenomenological indicator of degradation.

The Fourier transform used in the final stage of calculations is very sensitive to baseline subtraction $[8,9]$. A baseline was determined by means of smoothing (filtering of the noise of interest) using the moving average. Other smoothing methods based on polynomial averaging resulted in noticeable "quasiperiodical artifacts". Initial signal with drift and the noise after subtraction of the baseline are shown in the Figure 1.

Another possible source of artifacts is the limited length of the recorded periodogram. The windowing function is usually used. However, the usage of wrong windowing function can produce quite distorted and in some cases unreliable results in electrochemical noise analysis. That is why our calculations were carried out without any window function. To make it possible the method of Fourier transform was modified. The frequencies for analysis were selected in such a way that they made up an integer number of periods in the array of recorded noise data points. It was the key feature in calculations to avoid usage of any windowing function.

On the basis of electrochemical impedance data the Faradic resistance $R_{f}$ was obtained. It was calculated from low-frequency semicircle on a Nyquist plot. The Faradic admittance was calculated as its inverse value to compare it with load currents of a fuel cell.

\section{Results and Discussion}

A high frequency spectrum calculated for one sample of a fuel cell during its aging is presented in the Figure 2. In the Figure 3, load currents of a fuel cell at $400 \mathrm{mV}$ are compared with the amplitude of spectral maximum at $2.5 \mathrm{~Hz}$ frequency. The baseline was subtracted by applying 500 points moving average [10]. A spectral peak diminishes along with a decrease in load currents after each stage of aging. 

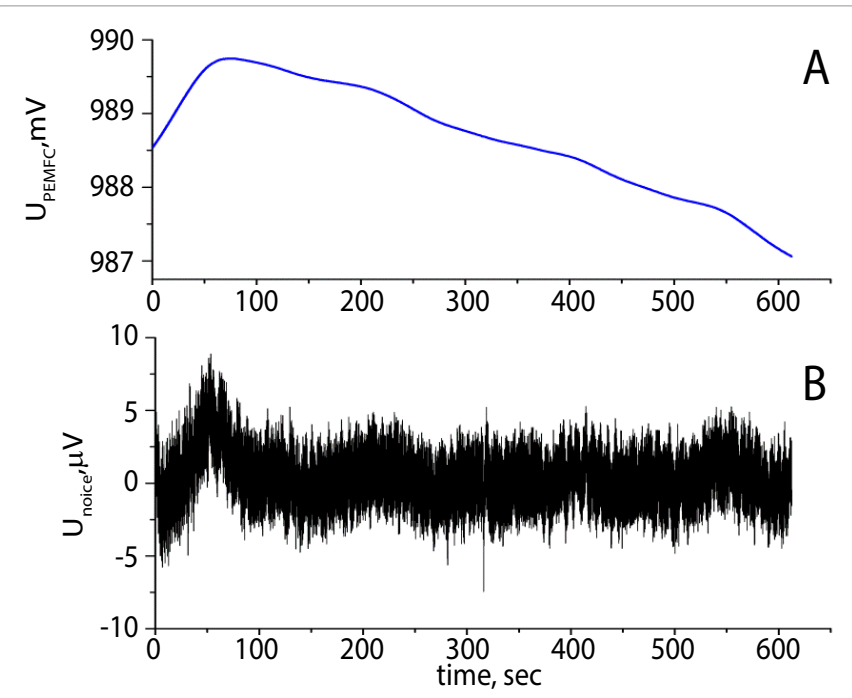

Figure 1: Electrochemical noise data before $(A)$ and after $(B)$ applying base line elimination procedure: $A$ - voltage between PEMFC electrodes, i.e., drifting baseline plus electrochemical noise; $\mathrm{B}$ - the electrochemical noise after moving average baseline subtraction.

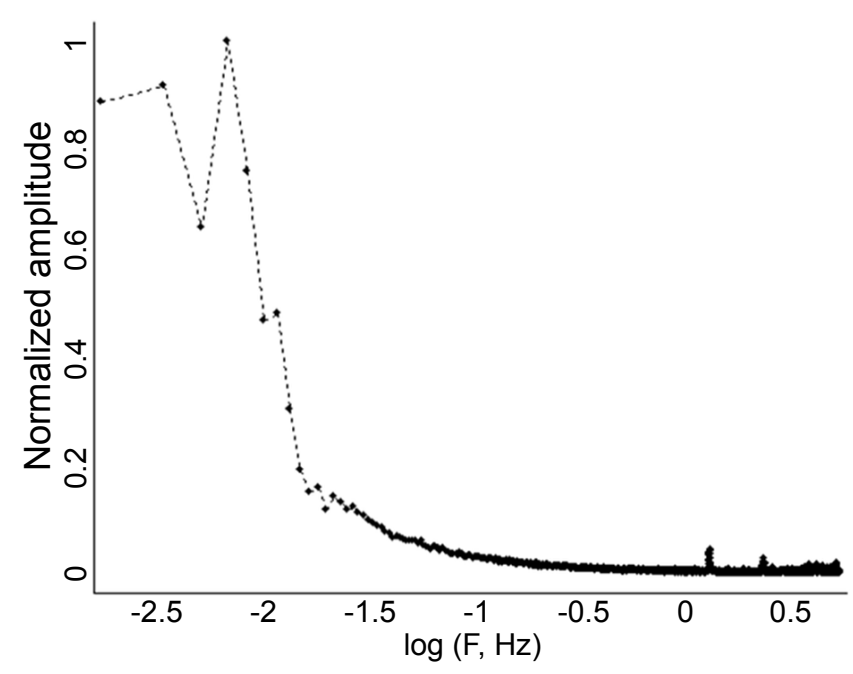

Figure 2: Noise spectrum obtained by Fourier transform after moving average baseline subtraction.

Like most fresh fuel cell, its load current rises after the first stage of aging treatment. And the same situation is observed for spectral maximum: its amplitude increases after the first degradation stage and then decreases (as the current does) after subsequent stages of degradation.

As seen from all diagrams in the Figure 3, Faradic admittance does not show any behavior correlated to the load currents of a fuel cell. Possibly this is caused by the fact that the Faradic admittance calculated for such a weakly aged fuel cell and measured at open circuit voltage should not strongly correspond to predicted load currents of a fuel cell in contrast to the results of impedance measurements for hardly aged fuel cell at voltage loads close to maximum power operation conditions [2].

In the case of separate electrodes in liquid electrolyte the power spectral density $S(f)$ was calculated as (1) from detrended electrochemical noise. This spectral density changes in a wide range for each sample and almost does not depend on conditions of measurements. However, the value of exponent $n$ in relation (2) is influenced to some extent by experiment conditions.

In contrast to electrodes in FC, a correlation of $n$ and aging is also observed. As the spectral power decreases for all frequencies, a drop of amplitude in low-frequency part of spectrum makes up the main contribution to the decrease in fluctuations power. That is why the degradation leads to the decrease in exponent $n$. Thus, in the log-log power spectrums, there is a decrease of the slope of approximation line (Figure 4). A significant growth of exponent after the first degradation stage correlates with the rise of cyclic voltammetry current for these electrodes (Figure 5). Probably, it is a consequence of an activation of electrodes at the first aging stage that exceeds slow degradation.

In general, degradation can be described as a diffusion-controlled process. The exponent $n$ characterizes the long-term memory or fractal diffusion $[11,12]$. Fick's diffusion corresponds to $n=2$. For lower $n$, the main contribution is made by the processes of limited sizes. One can assume that these spectral changes correspond to the dissolving of active catalyst particles of minimum size. In this case, a decrease in the exponent $n$ corresponds to the growth of metal clusters accompanied by the reduction of total catalytic activity [13].

\section{Conclusions}

Electrochemical noise of a polymer electrolyte fuel cell was measured and analyzed. Baseline subtraction by the moving average method was found favorable for obtaining better results than in the case of polynomial subtraction because the last one can add some resonant artifacts. An accurate baseline subtraction made it possible to utilize a Fourier transform for the analysis of electrochemical noise of such electrochemical power source as a PEM fuel cell.

Integer number of periods for Fourier transform frequency was shown to produce stable results for characteristic frequencies of electrochemical power source noise spectra without applying windowing functions.

The amplitude of spectral maximums was shown to reduce with the decrease in load current of a fuel cell during its aging. These calculations demonstrate better results in respect to load currents than data of electrochemical impedance analysis.

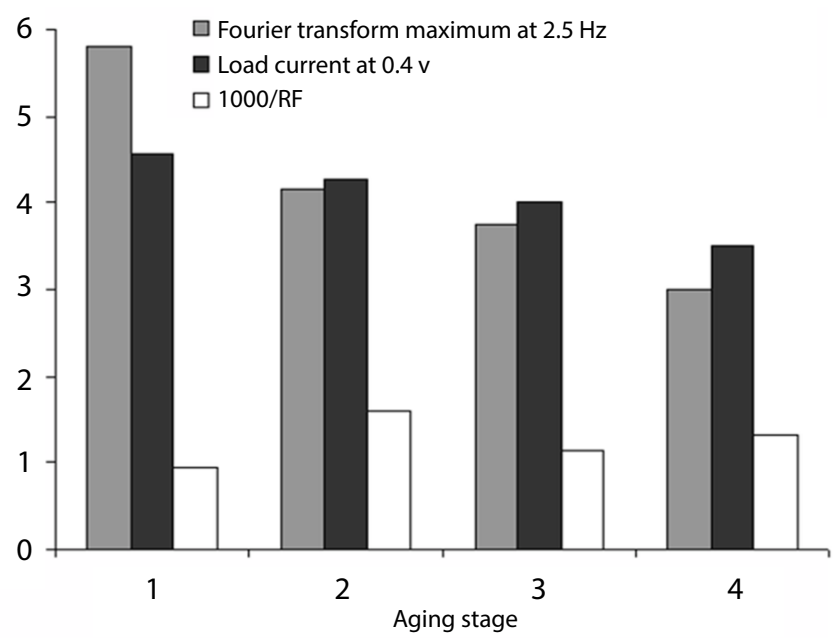

Figure 3: Spectral maximum at $2.5 \mathrm{~Hz}$ of moving average baseline subtracted electrochemical noise, load currents (divided by 100) of fuel cell at $0.4 \mathrm{~V}$ and Faradic admittance (multiplied by 1000) at different stages of aging of a fuel cell. 


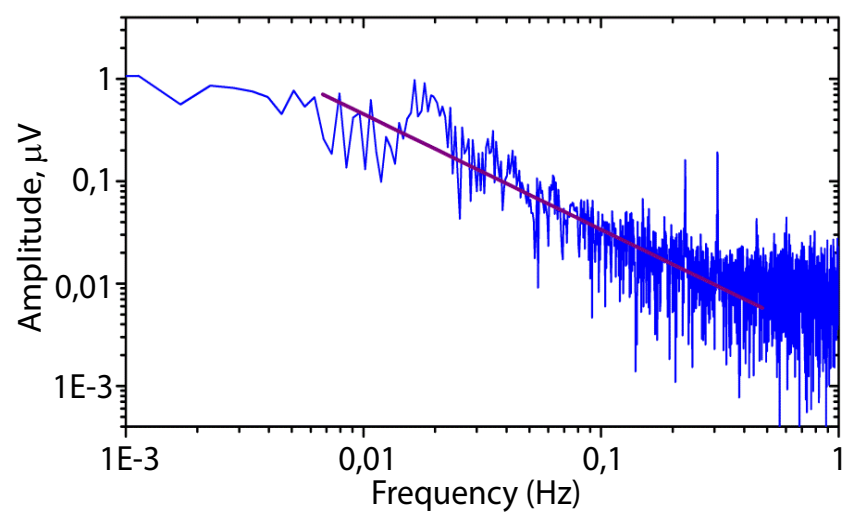

Figure 4: The spectrum of electrochemical noise of separate electrodes calculated for a wide frequency range. The purple line represents a linear regression in the range of $0.006-0.5 \mathrm{~Hz}$, its power $n=1.13$.

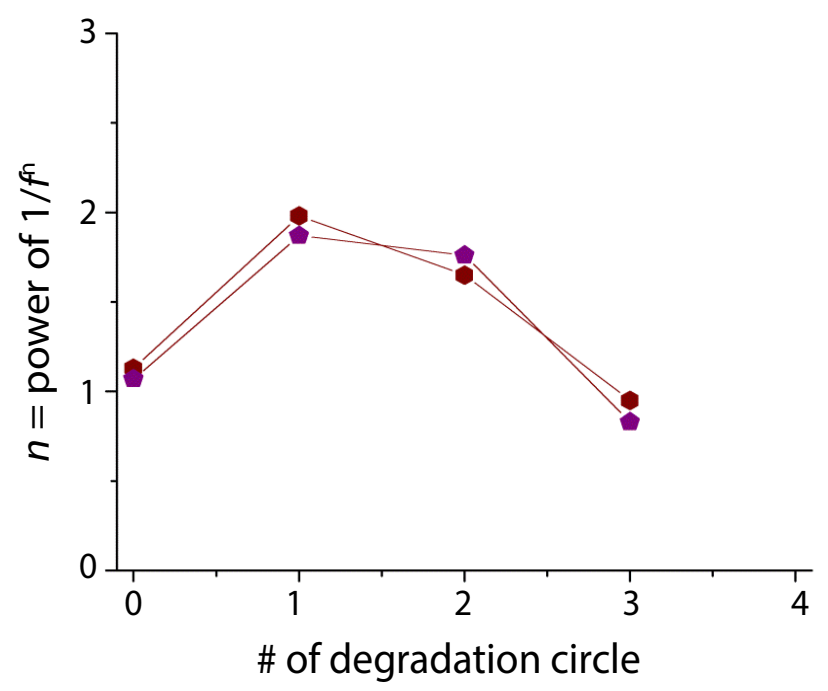

Figure 5: The value of spectral exponent of flicker noise of separate electrodes (two different samples) vs degradation stage.

The degradation of electrodes with electrocatalyst for a fuel cell leads to regular changes in the spectral exponent of flicker-noise. This exponent in frequency dependence (i.e., slope of the power spectrum) is the reproducible characteristic governed by electrochemical processes at the electrode. Thus, the fast degradation of an electrode results in the decrease in spectral exponent, meanwhile there is no dependence on noise amplitude.

On the contrary, load currents of a fuel cell increased after the first step of fast degradation achieved by cyclic voltammetry (in a wide potential range). It may be caused by an activation of catalytic surface. Remarkably, this phenomenon corresponds to the growth of spectral peak amplitude at $2.5 \mathrm{~Hz}$ and the increase in slope of the frequency dependence of flicker noise.

\section{Acknowledgements}

This work was supported by the Ministry of Education of Russian Federation. Project number is 14.604.21.0087 and ident is RFMEFI60414X0087.

\section{References}

1. Astafev EA, Dobrovolsky YA (2007) Behavior of membrane-electrode assemblages of polymer electrolyte fuel cells: electrochemical methods of investigation of electrocatalytical activity and corrosion stability of electrodes. Int Sci J Alternative Energy and Ecology 12: 21-27.

2. Astafev EA, Lyscov NV, Gerasimova EV (2009) Electrochemical methods of investigation of cathodes of fuel cell with solid electrolyte. Int Sci J Alternative Energy and Ecology 8: 55-69.

3. Liu L (2008) Pitting mechanism on an austenite stainless steel nanocrystalline coating investigates by electrochemical noise and in-situ AFM analysis. Electrochim Acta 54: 768-780.

4. Grafov BM (2007) Theory of equilibrium bispectra of nonlinear multiports: A langevin approach. Russ J Electrochem 43: 108-112.

5. Klyuev AL, Grafov BM, Dobrovol'skii YA, Davydov AD, Ukshe AE (2015) Variability of Discrete Chebyshev Spectra of Electrochemical Noise. Russ J Electrochem 51: 1180-1185.

6. Timashev SF, Polyakov YS (2007) Review of flicker noise spectroscopy in electro-chemistry. Fluctuation and Noise Letters 7: R15-R47.

7. Ukshe A, Chub A, Astafiev E, Dobrovolsky Y (2014) Investigation on Electrochemical Noise in the Cell with Advanced Superionic. Joint 12th Russia/CIS/baltic/Japan Symposium on Ferroelectricity and 9th Interbational Confertence Functional Materials and Nanotechnologies "RCBJSF-2014FM\&NT", September 29-October 2, 2014, Riga, Latvia.

8. Grafov BM, Dobrovol'skii YA, Davydov AD, Ukshe AE, Klyuev AL, et al. (2015) Electrochemical Noise Diagnostics: Analysis of Algorithm of Orthogonal Expansions. Russ J Electrochem 51: 503-507.

9. Sia DH, Behnamian $Y(2015)$ Ways to noise elimination from the $D C$ current at electrochemical noise measurement. Russ J Electrochem 51: 675-684.

10. Chatfield C (1989) The Analysis of Time Series. An Introduction to 4th edn Chapman and Hall, London, pp: 94-95.

11. Das S (2011) Functional Fractional Calculus. Springer, p: 598.

12. Mandelbrot BB (1999) Multifractals and $1 / f$ Noise: Wild Self-Affinity in Physics. Springer, p: 442.

13. Zyubin AS, Zyubina TS, Dobrovol'skii YA, Volokhov VM, Bazhanova ZG (2011) Activation of Molecular Hydrogen on Platinum Nanoparticles: QuantumChemical Modeling. Russ J Inorg Chem 56: 1290-1300. 